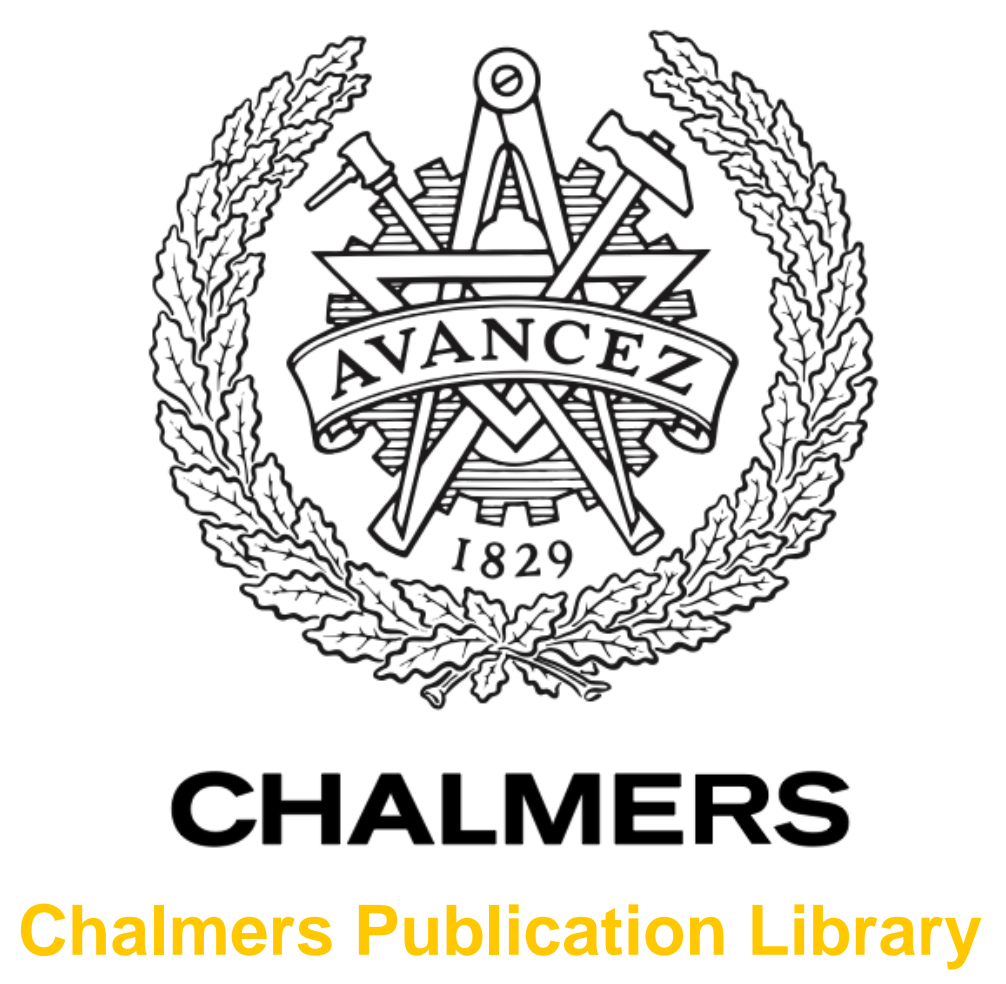

Inverse modelling of GNSS multipath for sea level measurements - initial results

This document has been downloaded from Chalmers Publication Library (CPL). It is the author's version of a work that was accepted for publication in:

Proceedings of the 2016 IEEE International Geoscience and Remote Sensing Symposium (IGARSS 2016)

Citation for the published paper:

Strandberg, J. ; Hobiger, T. ; Haas, R. (2016) "Inverse modelling of GNSS multipath for sea level measurements - initial results". Proceedings of the 2016 IEEE International Geoscience and Remote Sensing Symposium (IGARSS 2016)

Downloaded from: http://publications.lib.chalmers.se/publication/242356

Notice: Changes introduced as a result of publishing processes such as copy-editing and formatting may not be reflected in this document. For a definitive version of this work, please refer to the published source. Please note that access to the published version might require a subscription. 


\title{
INVERSE MODELLING OF GNSS MULTIPATH FOR SEA LEVEL MEASUREMENTS - INITIAL RESULTS
}

\author{
Joakim Strandberg, Thomas Hobiger, and Rüdiger Haas \\ Chalmers University of Technology \\ Department of Earth and Space Sciences \\ SE-412 96, Gothenburg, Sweden.
}

\begin{abstract}
We present a new method to retrieve sea level from GNSS SNR data that relies upon inverse modelling of the detrended SNR. This method can simultaneously use data from both GPS and GLONASS, and both L1 and L2 frequencies, to improve the solution with respect to prior studies. Results from the GNSS$\mathrm{R}$ installation at Onsala Space Observatory are presented and the retrieved sea level heights are compared with a co-located pressure mareograph. The method is found to give an RMS error of $1.8 \mathrm{~cm}$. The results are also compared against previous implementations of GNSS tide gauges and found to have lower RMS than both the earlier SNR algorithm and also the dual receiver, phase delay method.
\end{abstract}

Index Terms - GNSS-R, Inverse modelling, Reflectometry, Sea level, Tide gauge

\section{INTRODUCTION}

The idea that sea level measurements could be done passively using available GNSS signals was proposed already over two decades ago [1]. Since then several methods of using GNSS signals for measuring sea level has been proposed, using various degrees of specialized equipment. The first conceived ground based GNSS-R tide gauges use two receivers; one with upward antenna looking receiving primarily direct signals, and one with downward looking antenna receiving the reflected signal. Using the difference in time delay between the signals received by the two receivers, the height of the reflector surface can be calculated.

However, it has also been shown that geodetic-class offthe-shelf GNSS receivers can be used for sea height retrieval without modification [2], using the signal to noise ratio (SNR) of the GNSS satellites. Such stations are already installed on many coastal sites, and have been so for some time, and therefore their data could be used for calculating the sea level for several years back in time. A drawback of this one-receiver approach is its lower time resolution [2], but at the same time the operation has been shown to be more reliable in high wind conditions than two-receiver installations [3].

\section{ONSALA GNSS-R INSTALLATION}

The GNSS-R tide gauge at the Onsala Space Observatory was installed in fall of 2011, and has been previously described [2]. The equipment at the site can perform both single and dual receiver operation. During the time from which the GNSS data were collected a pressure mareograph, with a nominal uncertainty of $5 \mathrm{~mm}$, were available $10 \mathrm{~m}$ from the GNSS-R station, which is used as a reference for the GNSS-R tide gauge implementations.

\section{GNSS TIDE GAUGE}

The SNR GNSS tide gauge builds upon using multipath effects in GNSS signals to derive the sea height in the vicinity of the GNSS receiver. The SNR of GNSS signals, which are affected by interference from reflections, is dependent on the elevation angle to the satellite. Therefore, as the satellite travels along its arc, the SNR will create a characteristic oscillating pattern overlaid on a long time trend [4].

The frequency of these oscillations depend on the height, $h$, between the antenna and the reflecting surface. Removing the trend from the SNR, the remainder can be modelled as [5]:

$$
\delta \mathrm{SNR}=A \cos \left(\frac{4 \pi h}{\lambda} \sin \varepsilon+\varphi\right) \mathrm{e}^{-K \sin ^{2} \varepsilon} .
$$

Here, $\varepsilon$ is the angle to the satellite measured from the horizon.

Noting that $\delta$ SNR can be rewritten as a function of $x=$ $\sin (\varepsilon)$, previous efforts on SNR tide gauges have focused on using spectrum analysis to find the main frequency in the individual $\delta$ SNR arcs, which corresponds to $\frac{4 \pi h}{\lambda}$. Since the function is unevenly sampled in $x$, Fast Fourier transform will not work, and an algorithm such as Lomb-Scargle must be used to retrieve the power spectrum. In the original form this assumes a stationary reflector surface for the whole arc. For example this is reasonable in the case of snow height measurements [6] and sea level measurements where the tidal ranges are low [2]. For sites where the tidal range is too high this model is too inaccurate since the reflector height changes significantly during the arc, which introduces systematic error 
depending on if the reflector height is rising or falling. To compensate for this, some work has been done to provide a correction term for the height change of the surface, which accounts for a linear change in the reflector height [3].

\subsection{Inverse modelling of GNSS SNR data}

In the proposed method, the height is retrieved through inverse modelling the $\delta$ SNR by fitting a curve to it. Figure 1 depicts the $\delta$ SNR from one arc, and a fitted function using the process described below. A similar procedure has been previously used to measure snow height [7], however in that method the reflector height was assumed to be constant, and only one measurement was derived for each arc. In this work we will model height as a time dependent function to account for the changes in $\delta \mathrm{SNR}$-frequency that occurs when measuring changing water levels.

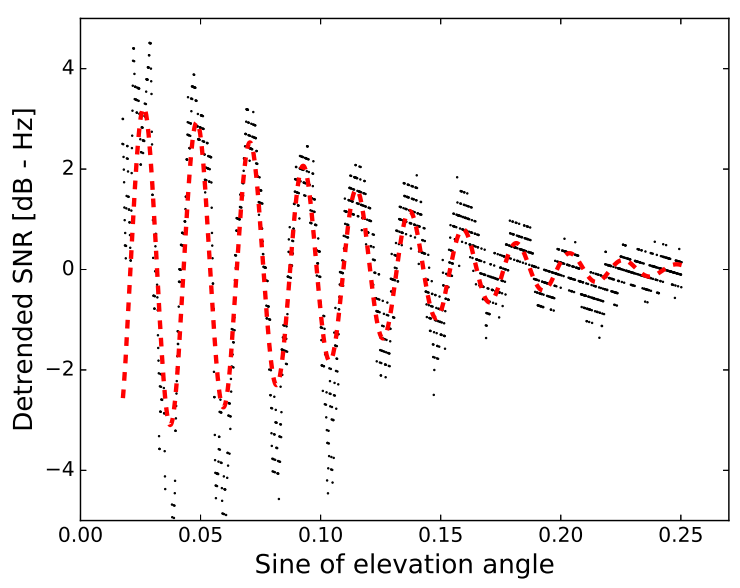

Fig. 1. Detrended SNR from one arc (black), together with the modelled $\delta \mathrm{SNR}$ (red).

From Equation (1) we see that we can model the $\delta$ SNR using only four parameters: the amplitude $A$, a phase offset $\varphi$, a damping factor $K$, and the height $h$. Since $\varepsilon$ in Equation (1) is a function of time, we can also introduce time dependent height $h(t)$, and use $t$ as our variable instead of $x=\sin (\varepsilon)$. This allows the height of the reflector surface, and therefore frequency of the $\delta \mathrm{SNR}$, to change during the course of one arc. Furthermore, instead of one single measurement of the sea level per satellite arc the method gives a continuous function for the height.

The height is modelled as a smooth function using a 2 nd order b-spline basis. The b-spline basis of degree $r$ is defined recursively using the node-points $t_{0}, \ldots, t_{n}$ as [8]:

$$
\begin{gathered}
N_{j}^{0}(t)= \begin{cases}1 & \text { if } t_{j} \leq t \leq t_{j+1} \\
0 & \text { otherwise }\end{cases} \\
N_{j}^{r}(t)=\frac{t-t_{j}}{t_{j+r}-t_{j}} N_{j}^{r-1}(t)+\frac{t_{j+r+1}-t}{t_{j+r+1}-t_{j+1}} N_{j+1}^{r-1}(t) .
\end{gathered}
$$

With these basis functions the height function is approximated using the node heights $h_{0}, \ldots, h_{n}$ :

$$
\hat{h}(t)=\sum_{j} h_{j} N_{j}^{r}(t) .
$$

Data from three consecutive days are used for fitting the height function and the other parameters. However, only the data from the middle day is used as the final solution. The underlying fitting problem becomes highly non-linear because of the form of Equation (1), and is solved iteratively using numerical methods.

As already mentioned, our approach is able to use all arcs during a chosen timespan to fit a continuous height function. Worth noting is that this is not confined to arcs from a specific GNSS system, or a specific frequency. It is possible to use all available signals simultaneously, thereby increasing the amount of data available for the fitting.

\section{RESULTS AND COMPARISON}

The number of nodes chosen for the b-spline approximation of the height is important for a proper modelling of site specific reflector height variations. If too few nodes are chosen, the resulting height-function will not be able to resolve all tides, but if too many nodes are used the function will instead be to sensitive to outliers in the data set. For this implementation 37 evenly spaced nodes, i.e. a two hour separation, are used for the whole three days that are processed simultaneously. As shown in Figure 2, this gives the height function enough resolution to resolve all but the most short time scale behaviour.

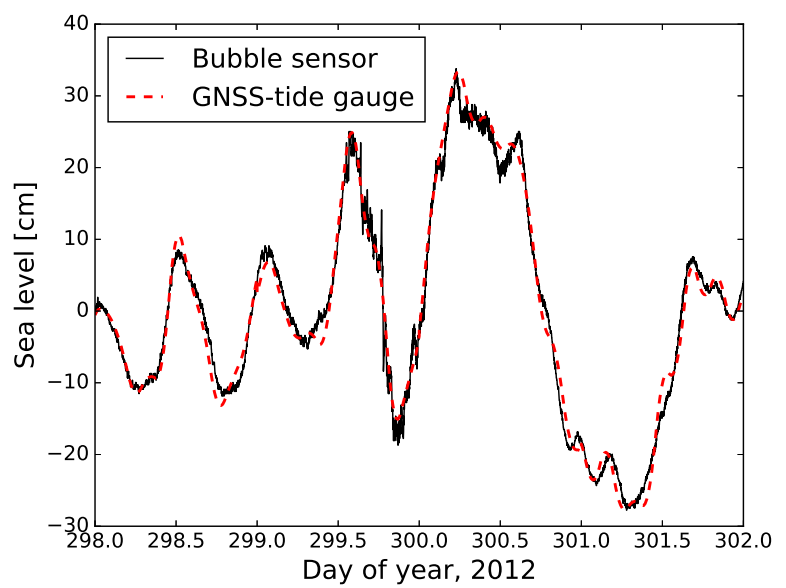

Fig. 2. Sea level derived from inverse modelling the detrended SNR, and the reference data from the on site mareograph for a subset of the data used for validation. Since the mareograph and the GNSS solution do not have the same reference level, the mean of the two data sets are removed before plotting.

The derived sea level results were compared against the 
Table 1. RMS difference and correlation between three different GNSS-R tide gauge implementations and the reference mareograph at Onsala from doy 273 to doy 303, year 2012. The result of the Lomb-Scargle and the phase delay methods are the best results gained for the respective method in the paper by [3].

\begin{tabular}{|c|c|c|c|c|c|}
\hline & \multicolumn{4}{|c|}{ Single receiver (SNR) } & \multirow{3}{*}{$\begin{array}{l}\frac{\text { Dual receiver }}{\text { Phase delay [3] }} \\
\text { GLONASS, L2 }\end{array}$} \\
\hline & \multicolumn{3}{|c|}{ Inverse modelling (this paper) } & \multirow{2}{*}{$\frac{\text { Lomb-Scargle [3] }}{\text { GPS, L1 }}$} & \\
\hline & GPS+GLONASS & GPS & GLONASS & & \\
\hline Correlation: & 0.99 & 0.99 & 0.99 & 0.97 & 0.96 \\
\hline RMS error $[\mathrm{cm}]$ : & 1.8 & 2.0 & 2.9 & 4.0 & 3.2 \\
\hline Mean diff. $[\mathrm{cm}]$ : & 1.5 & 1.6 & 2.3 & 3.1 & 2.3 \\
\hline
\end{tabular}

co-located pressure sensor by evaluating the final b-spline function for the height at the time of all pressure sensor measurements. For comparison, the chosen data set has previously been used for the Lomb-Scargle method and phase delay method by [3]. The set is taken between day of year 273 and 303 in the year 2012. Since the GNSS-R tide gauge and the pressure sensor measure with respect to different offsets, the mean values of each time series are removed before the comparison.

The results of the comparison are summarized in Table 1, where the inverse modelling method is compared against the best results of the Lomb-Scargle algorithm and phase delay method. As can be seen from the table, the inverse modelling represents a significant improvement in performance for the single receiver operation, reducing the RMS error by more than $50 \%$ and decreasing the mean difference to only $1.5 \mathrm{~cm}$.

\section{CONCLUSIONS}

Inverse modelling for sea level retrieval has a potential to increase the precision of GNSS-R tide gauges. It even outperforms the current dual receiver phase-delay method, without the need for specialized equipment. This means that potentially all GNSS receivers already installed near open water can be used to retrieve sea level. Furthermore, since the method is based on SNR analysis, it can continue to operate during high winds, in which the phase delay algorithm fails to lock on the satellites with the nadir looking antenna. This leads to a more stable and reliable operation.

Another advantage of this new method is its high temporal resolution; sea level values can be obtained at any time resolution depending only on the choice of the b-spline nodes. This is in contrast to the sparsely sampled arc-wise solutions, with a mean spacing of roughly half an hour, which earlier SNR methods give.

The ability to use data from different GNSS systems is also seen to increase the performance, further reducing the RMS. Therefore, it is of interest to add other systems, such as GALILEO and COMPASS, in the future.

However, to verify these results, the algorithm needs to be tested on more GNSS installations and sites with varying tidal behaviour and range.

\section{REFERENCES}

[1] M. Martin-Neira, "A passive reflectometry and interferometry system (PARIS): Application to ocean altimetry," ESA journal, vol. 17, pp. 331-355, 1993.

[2] K. M. Larson, J. S. Löfgren, and R. Haas, "Coastal sea level measurements using a single geodetic GPS receiver," Advances in Space Research, vol. 51, no. 8, pp. 13011310, 2013.

[3] J. S. Löfgren and R. Haas, "Sea level measurements using multi-frequency GPS and GLONASS observations," EURASIP Journal on Advances in Signal Processing, vol. 2014, no. 1, pp. 1-13, 2014.

[4] K. M. Larson, E. E. Small, E. D. Gutmann, A. Bilich, P. Axelrad, and J. J. Braun, "Using GPS multipath to measure soil moisture fluctuations: initial results," GPS solutions, vol. 12, no. 3, pp. 173-177, 2008.

[5] F. G. Nievinski and K. M. Larson, "Forward modeling of GPS multipath for near-surface reflectometry and positioning applications," GPS solutions, vol. 18, no. 2, pp. 309-322, 2014.

[6] K. M. Larson, E. D. Gutmann, V. U. Zavorotny, J. J. Braun, M. W. Williams, and F. G. Nievinski, "Can we measure snow depth with GPS receivers?, Geophysical Research Letters, vol. 36, no. 17, 2009.

[7] F. G. Nievinski and K. M. Larson, "Inverse modeling of GPS multipath for snow depth estimation-part II: application and validation," Geoscience and Remote Sensing, IEEE Transactions on, vol. 52, no. 10, pp. 6564-6573, 2014.

[8] E. J. Stollnitz, T. D. DeRose, and D. H. Salesin, "Wavelets for computer graphics: a primer. 1," Computer Graphics and Applications, IEEE, vol. 15, no. 3, pp. 76-84, 1995. 